\title{
Evolução ponderal na gravidez, preditores e consequências: estudo retrospetivo
}

Joana Sequeira, ${ }^{*}$ Carla Simões, ${ }^{* *}$ Verónica Colaço, ${ }^{* * *}$ Joana Dias****

\section{RESUMO}

Objetivos: Avaliar o aumento ponderal durante a gravidez e verificar se existe associação entre o aumento de peso na gravidez e o tipo de parto, paridade, complicações obstétricas e peso do recém-nascido.

Tipo de estudo: Estudo de coorte histórica.

Local: Centro de Saúde de Vagos, Agrupamento de Centros de Saúde Baixo Vouga II.

População: Grávidas vigiadas na unidade de saúde.

Métodos: Amostra constituída por mulheres que estiveram grávidas entre 2003 e 2010. As variáveis estudadas foram: idade, paridade, índice de massa corporal (IMC) pré-concecional, aumento ponderal, tipo de parto, complicações obstétricas e peso do recém-nascido. Para análise estatística utilizaram-se os testes de Qui-quadrado, ANOVA e Least Significant Difference.

Resultados: Foram estudadas 495 grávidas com idade média de 27 anos. No início da gravidez 35\% tinham IMC $\geq 25$, no final $46 \%$ tiveram aumento de peso superior ao recomendado. As grávidas com aumento de peso superior ao recomendado apresentaram em média um IMC pré-concecional significativamente maior que as grávidas com aumento de peso adequado ou inferior ao recomendado $(p<0,001)$. Os recém-nascidos filhos de grávidas com aumento de peso superior ao recomendado apresentaram um peso médio significativamente superior aos restantes recém-nascidos $(p=0,024)$. Não houve associação entre aumento de peso e tipo de parto, complicações obstétricas ou paridade.

Conclusões: Cerca de um terço das grávidas apresentaram excesso de peso ou obesidade no início da gravidez. A maioria das grávidas teve um aumento de peso superior ao recomendado para o seu IMC. As grávidas com maior IMC pré-concecional têm mais frequentemente ganho ponderal superior ao recomendado e têm filhos, em média, mais pesados. São necessários estudos para validar recomendações de aumento ponderal na gravidez para a população portuguesa.

Palavras chave: Gravidez; Obesidade; Aumento Ponderal.

\section{INTRODUÇÃO}

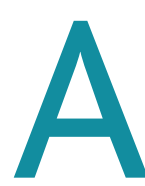
obesidade tornou-se nos últimos anos um grave problema de saúde pública, com um aumento progressivo da sua prevalência a nível mundial. ${ }^{1}$ A prevalência de obesidade na mulher em idade fértil, em tempos uma condição rara, tem aumentado drasticamente nos últimos anos. Um estudo realizado em 2009 pela Sociedade Portuguesa de

\footnotetext{
*Interna de Medicina Geral e Familiar USF Flor de Sal - Aces Baixo Vouga II **Interna de Medicina Geral e Familiar USF Flor de Sal - Aces Baixo Vouga II ***Assistente de Medicina Geral e Familiar USF Flor de Sal - Aces Baixo Vouga II ****Assistente graduada de Medicina Geral e Familiar USF Flor de Sal - Aces Baixo Vouga II
}

Ciências da Nutrição e Alimentação refere uma prevalência de pré-obesidade nas mulheres de $27,8 \%$ e de obesidade de $10,4 \% .^{2}$

A obesidade na mulher grávida aumenta o risco de complicações obstétricas e perinatais, tais como diabetes gestacional, hipertensão arterial, pré-eclâmpsia ou atraso de crescimento intrauterino. ${ }^{3,4,5}$ Mulheres com aumento de peso excessivo durante a gravidez têm também maior risco de retenção de peso pós-parto ou obesidade pós-parto. ${ }^{3,4} \mathrm{O}$ aumento de peso excessivo durante a gravidez também aumenta a frequência de eventos adversos para a criança.,

Um dos mais importantes indicadores do ganho de peso gestacional e do seu impacto na saúde da mãe e do filho é o peso pré-concecional ou o peso da grávida 
no início da gravidez. ${ }^{4} \mathrm{Em}$ 2009, o Institute of Medicine (IOM) publicou uma revisão das recomendações para o ganho de peso gestacional, de acordo com o índice de massa corporal (IMC) pré-concecional, publicadas pela primeira vez em 1990. Segundo estas recomendações, as mulheres com IMC menor que 18,5 devem ter um ganho ponderal entre 12,5 e $18 \mathrm{~kg}$; as mulheres com IMC entre 18,5 e 24,9 devem ter um ganho ponderal entre 11,5 e $16 \mathrm{~kg}$; as mulheres com IMC entre 25 e 29,9 devem ter um ganho ponderal entre 7,0 e 11,5 kg e as mulheres com IMC superior a 30 devem ter um ganho ponderal entre 5,0 e 9,0 kg.,6

Não foram encontrados estudos em Portugal referentes ao aumento de peso durante a gravidez. Tendo em conta as comorbilidades associadas e os elevados custos em saúde que condicionam, é essencial estudar a prevalência e as consequências do aumento ponderal excessivo durante a gravidez.

Os objetivos deste trabalho foram avaliar o aumento ponderal das grávidas seguidas no Centro de Saúde de Vagos entre 2003 e 2010 e verificar se existe alguma associação entre o aumento de peso na gravidez e o IMC pré-concecional, paridade, tipo de parto, complicações obstétricas e peso do recém-nascido (RN).

\section{MÉTODOS}

Realizamos um estudo retrospetivo e analítico tendo como população todas as mulheres inscritas no Centro de Saúde de Vagos que estiveram grávidas entre janeiro de 2003 e dezembro de 2010. Foram excluídas: grávidas não vigiadas no Centro de Saúde até às 36 semanas; gravidezes gemelares, gravidezes que resultaram em aborto e partos pré-termo. Recolhemos os dados através de consulta dos processos clínicos (informático e/ou papel). Os dados recolhidos foram: ano do parto, idade materna, paridade, pesos no início e no final da gravidez, altura, existência de complicações obstétricas, tipo de parto e peso do RN.

O aumento de peso na gravidez foi calculado pela diferença entre o peso final e inicial. O peso inicial foi o peso reportado pela grávida como sendo o peso anterior à gravidez. Na ausência desta informação foi considerado o peso da primeira consulta, sendo que todas as primeiras consultas ocorreram antes das 12 semanas. O peso final foi o peso registado na última consulta antes do parto, sendo esta depois das 36 semanas de ges- tação, inclusive. Consideramos três classes de aumento de peso (adequado, maior que o recomendado e menor que o recomendado) de acordo com os valores recomendados pelo IOM para o respetivo IMC. Consideramos as seguintes complicações obstétricas: diabetes gestacional (DG), hipertensão arterial (HTA), préeclâmpsia e atraso de crescimento intrauterino (ACIU). O parto foi considerado eutócico ou distócico, incluindo-se neste último os partos por cesariana, ventosa e/ou fórceps. Quanto ao peso do RN, consideramos três classes: baixo peso (inferior a $2.500 \mathrm{~g}$ ); normal (entre 2.500 e $4.000 \mathrm{~g}$ ) e macrossómico (superior a $4.000 \mathrm{~g}$ ).

Os dados obtidos foram analisados através do software SPSS ${ }^{\circledast}$ (Statistical Package for Social Sciences) versão 18.0. As variáveis quantitativas foram caraterizadas através da média, máximo, mínimo e desvio-padrão e as qualitativas recorrendo às frequências absolutas e relativas. Para comparação das grávidas com aumento de peso adequado, menor ou maior que o recomendado utilizou-se ANOVA de fator único para as variáveis quantitativas e teste de Qui-Quadrado para as qualitativas. Para analisar as diferenças entre as categorias de aumento de peso utilizou-se o teste Least Significant Difference (LSD). Todos os testes foram avaliados ao nível de significância de 0,05.

\section{RESULTADOS}

No Centro de Saúde de Vagos, entre os anos de 2003 e 2010, registaram-se 545 mulheres grávidas, das quais 50 foram excluídas após aplicação dos critérios de exclusão. A amostra foi assim constituída por 495 grávidas, cujas idades se compreenderam entre $14 \mathrm{e} 44$ anos, sendo a média de 27,3 anos, com desvio padrão (DP) de $\pm 4,88$ anos. Das grávidas estudadas, $52,5 \%$ eram nulíparas.

Previamente à gravidez, a maioria das grávidas, 307 (62\%), apresentava um IMC pré-concecional normal, enquanto $35 \%$ apresentavam excesso de peso ou obesidade (122 com excesso de peso e 50 com obesidade). Apenas 16 (3\%) tinham baixo peso.

Ao longo dos 8 anos do estudo, houve uma média de 62 partos por ano $(D P \pm 9,1)$. O ano em que ocorreram menos partos foi 2004 (52 partos), e o ano em que ocorreram mais partos foi 2006 (78 partos).

Relativamente ao aumento de peso, a média do ganho ponderal foi $14,5 \mathrm{~kg}(D P \pm 6,04 \mathrm{~kg})$, com um míni- 


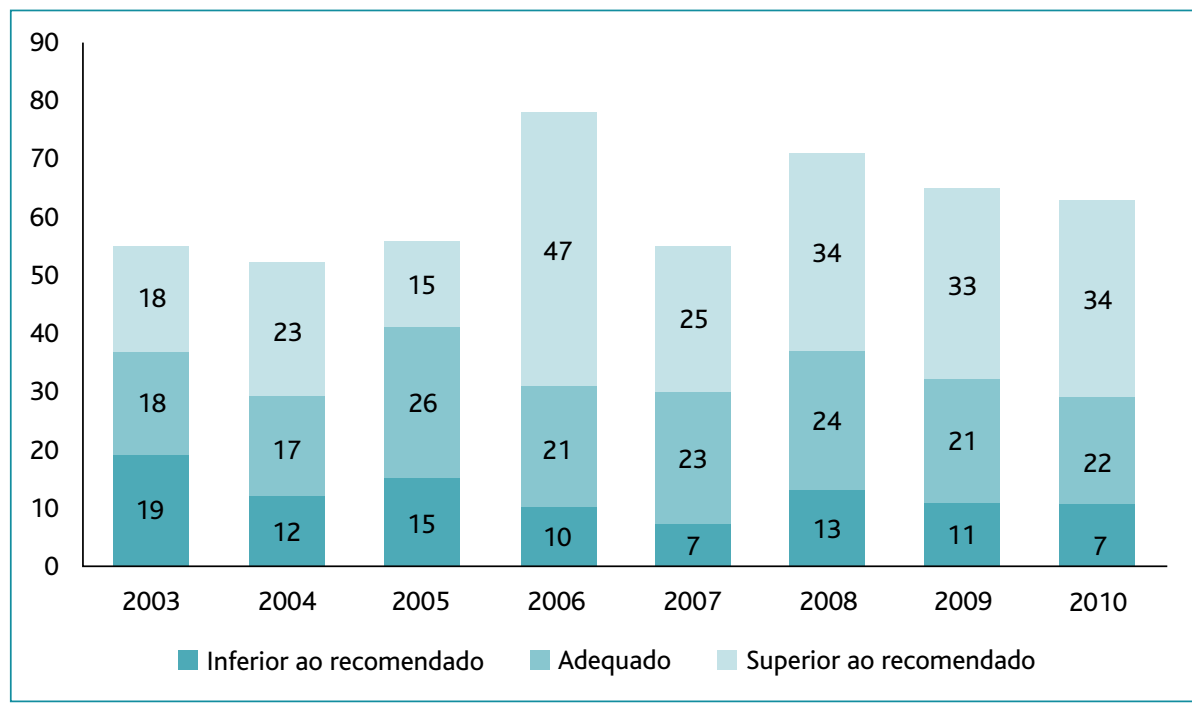

Figura 1. Distribuição das grávidas de acordo com o tipo de aumento de peso para o IMC pré-concecional, ao longo dos oito anos do estudo.

mo de -2,0 kg e um máximo de 31,0 kg. Quando analisamos o aumento de peso recomendado de acordo com o IMC pré-concecional, verificamos que $34,7 \%$ das grávidas tiveram um aumento de peso adequado ao seu IMC, 46,3\% aumentaram mais do que o recomendado e $19,0 \%$ tiveram um aumento de peso inferior ao recomendado. A figura 1 representa a distribuição de cada uma das classes de aumento de peso ao longo dos oito anos do estudo.

Registaram-se 37 complicações obstétricas (7,5\%):

\begin{tabular}{|c|c|c|c|c|c|c|}
\hline & & \multirow{2}{*}{$\begin{array}{l}\text { Total } \\
(n=495)\end{array}$} & \multicolumn{3}{|c|}{ Aumento ponderal } & \multirow[b]{2}{*}{$P$} \\
\hline & & & $\begin{array}{l}\text { Menor } \\
(n=94)\end{array}$ & $\begin{array}{l}\text { Adequado } \\
(\mathrm{n}=172)\end{array}$ & $\begin{array}{c}\text { Maior } \\
(n=229)\end{array}$ & \\
\hline $\begin{array}{l}\text { Complicações } \\
\text { [n (\%)] }\end{array}$ & $\begin{array}{l}\text { não } \\
\text { sim }\end{array}$ & $\begin{array}{r}458(92,5) \\
37(7,5)\end{array}$ & $\begin{array}{r}87(92,6) \\
7(7,4)\end{array}$ & $\begin{array}{r}160(93,0) \\
12(7,0)\end{array}$ & $\begin{array}{r}211(92,1) \\
18(7,9)\end{array}$ & 0,946 \\
\hline Parto [n (\%)] & $\begin{array}{l}\text { eutócico } \\
\text { distócico }\end{array}$ & $\begin{array}{l}245(49,5) \\
250(50,5)\end{array}$ & $\begin{array}{l}44(46,8) \\
50(53,2)\end{array}$ & $\begin{array}{l}95(55,2) \\
77(44,8)\end{array}$ & $\begin{array}{l}106(46,3) \\
123(53,7)\end{array}$ & 0,176 \\
\hline $\mathrm{RN}[\mathrm{n}(\%)]$ & $\begin{array}{l}\text { baixo peso } \\
\text { normal } \\
\text { macrossómico }\end{array}$ & $\begin{array}{r}22(4,4) \\
447(90,3) \\
26(5,3)\end{array}$ & $\begin{array}{r}6(6,4) \\
84(89,4) \\
4(4,2)\end{array}$ & $\begin{array}{r}8(4,6) \\
156(90,7) \\
8(4,6)\end{array}$ & $\begin{array}{r}8(3,5) \\
207(90,4) \\
14(6,1)\end{array}$ & 0,752 \\
\hline
\end{tabular}

$\mathrm{RN}$ : recém-nascido
21 casos de DG, 10 casos de HTA, 4 casos de pré-eclâmpsia e 2 casos de ACIU.

Quanto ao tipo de parto, $50,5 \%$ dos partos foram distócicos, tendo a maioria destes sido cesarianas (152; 30,7\% de todos os partos).

A média de peso dos $\mathrm{RN}$ foi $3.306,5 \mathrm{~g}(D P \pm 481,90 \mathrm{~g})$, o peso mínimo registado foi 2.065,0 g e o máximo foi $4.490,0$ g. Cerca de $90 \%$ dos RN apresentavam peso considerado normal (entre 2.500 e 4.000 g). A percentagem de $\mathrm{RN}$ macrossómicos e com baixo peso foi idêntica, correspondendo a cerca de $5 \%$.

O quadro I resume a distribuição das complicações e tipo de parto de acordo com as categorias de aumento ponderal durante a gravidez.

Observou-se uma associação estatisticamente significativa entre o aumento do peso e o IMC pré-concecional $(p<0,001)$. No grupo das grávidas obesas, $85,7 \%$ tiveram um aumento ponderal superior ao recomendado. Nas grávidas com excesso de peso esta proporção foi de $60,2 \%$. Nenhuma grávida obesa teve aumento de peso inferior ao recomendado, tendo sido essa percentagem de $15,4 \%$ nas grávidas com excesso de peso, $22,8 \%$ nas com IMC normal e $31,3 \%$ naquelas que eram magras. Relativamente às grávidas que tiveram um aumento ponderal entre os valores recomendados, $31,3 \%$ eram magras, $42,3 \%$ tinham peso normal, $24,4 \%$ tinham excesso de peso e $14,3 \%$ eram obesas (Quadro II).

Neste estudo o tipo de parto, a paridade e as complicações obstétricas foram independentes do ganho 
gestacional de peso.

Observando-se diferenças estatisticamente significativas (Quadro III) entre as categorias de aumento de peso para o IMC inicial $(p<0,001)$, realizou-se o teste de comparações múltiplas LSD para analisar entre que categorias existiam diferenças. Constatou-se que estas ocorriam entre as grávidas com ganho médio de peso inferior ao esperado e as com ganho médio de peso superior ao esperado (inferior $=23,1 \pm 2,7$ vs

QUADRO II. Características maternas prévias à gravidez segundo categorias de ganho ponderal.

\begin{tabular}{|c|c|c|c|c|c|c|}
\hline & & \multirow{2}{*}{$\begin{array}{c}\text { Total } \\
\text { (n=495) }\end{array}$} & \multicolumn{3}{|c|}{ Aumento ponderal } & \multirow[t]{2}{*}{$p$} \\
\hline & & & $\begin{array}{l}\text { Menor } \\
(n=94)\end{array}$ & $\begin{array}{l}\text { Adequado } \\
(n=172)\end{array}$ & $\begin{array}{c}\text { Maior } \\
(n=229)\end{array}$ & \\
\hline \multirow[t]{3}{*}{ Paridade [n (\%)] } & nulípara & $260(52,5)$ & $47(50,0)$ & $85(49,4)$ & $128(55,9)$ & 0,377 \\
\hline & multípara & $235(47,5)$ & $47(50,0)$ & $87(50,6)$ & $101(44,1)$ & \\
\hline & baixo peso & $16(3,2)$ & $5(5,3)$ & $5(2,9)$ & $6(2,6)$ & 0,001 \\
\hline \multirow{3}{*}{$\begin{array}{l}\text { IMC categorias } \\
{[\mathrm{n}(\%)]}\end{array}$} & normal & $307(62,0)$ & $70(74,5)$ & $130(75,6)$ & $107(46,7)$ & \\
\hline & $\begin{array}{l}\text { excesso de } \\
\text { peso }\end{array}$ & $123(24,8)$ & $19(20,2)$ & $30(17,4)$ & $74(32,3)$ & \\
\hline & obesidade & $49(9,9)$ & $0(0,0)$ & $7(4,1)$ & $42(18,3)$ & \\
\hline
\end{tabular}

IMC: índice de massa corporal superior $=25,7 \pm 4,6 ; p<0,001)$ e entre as grávidas com ganho de peso adequado e as com ganho de peso superior ao esperado (adequado $=22,9 \pm 3,6$ vs superior $=25,7 \pm 4,6 ; p<0.001$ ).

Observaram-se igualmente (Quadro III) diferenças estatisticamente significativas entre as categorias de acréscimo de peso e o peso do recém-nascido ( $p=$ $0,024)$. Constatou-se, com base no teste LSD, que estas ocorriam entre as grávidas com ganho médio de peso inferior ao esperado e as com ganho de peso superior ao esperado (inferior $=$ $3.217,0 \pm 437,1$ vs superior $=$ $3.366,0 \pm 487,4 ; p=0,011) \mathrm{e}$ entre as grávidas com ganho de peso adequado e as com ganho de peso superior ao esperado (adequado = $3.276,3 \pm 490,0$ vs superior $=$ $3.366,0 \pm 487,4 ; p=0,044)$.

\section{DISCUSSÃO}

A obesidade é um grave problema de saúde pública que tem aumentado na população em geral e inclusivamente na mulher em idade reprodutiva. ${ }^{1} \mathrm{Na}$ nossa amostra a prevalência de excesso de peso pré-concecio- nal foi de $20 \%$ e obesidade $10 \%$. Estes resultados são concordantes com outros estudos. ${ }^{7}$

Ao longo dos 8 anos do estudo, $46 \%$ das grávidas tiveram aumento de peso superior ao recomendado. Noutros estudos realizados no Canadá, Austrália e Estados Unidos da América, a percentagem de mulheres com ganho ponderal gestacional excessivo variou entre 36 e $50 \% .3,8,9$

Neste estudo concluímos que existe uma associação estatisticamente significativa entre a classe de aumen-

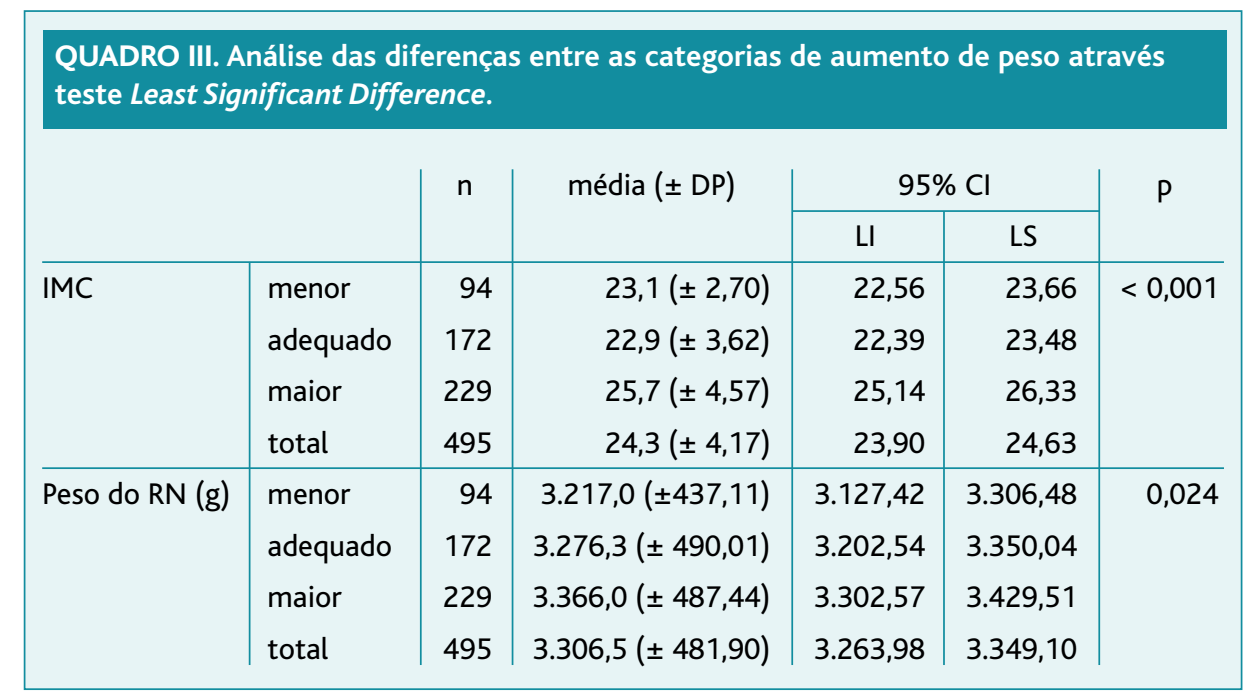

DP: desvio padrão; Cl: intervalo de confiança; LI: limite inferior; LS: limite superior; RN: recém-nascido; IMC: índice de massa corporal 
to de peso (adequado, maior que o recomendado e menor que o recomendado) e o IMC pré-concecional, ou seja, a percentagem de grávidas com aumento de peso superior ao recomendado foi significativamente maior nos grupos de grávidas com obesidade e excesso de peso do que nas grávidas que apresentavam IMC normal ou baixo peso no início da gravidez. Estes resultados estão de acordo com a maioria dos estudos que referem que as mulheres com excesso de peso ou obesidade têm maior risco de aumento de peso excessivo na gravidez do que as mulheres com IMC normal.,9

Neste estudo não foi possível verificar associação entre a classe de aumento de peso e o tipo de parto, paridade ou complicações obstétricas. Este facto pode dever-se, em parte, à pequena percentagem de complicações obstétricas detetada na nossa amostra $(7,5 \%)$, esta, por sua vez, muito provavelmente relacionada com os critérios de exclusão aplicados. No entanto, existe evidência que demonstra associação entre o excessivo aumento de peso na gravidez com maior risco de parto por cesariana e complicações obstétricas, nomeadamente DG e HTA gestacional., ${ }^{3,8,10,11}$ Relativamente à paridade, os estudos realizados verificaram que o aumento de peso na gravidez está inversamente associado à paridade. ${ }^{9,12} \mathrm{~A}$ justificação para estas diferenças poderá também estar relacionada, por um lado, com diferenças metodológicas, uma vez que os estudos referidos consideraram três categorias de paridade e neste estudo consideramos apenas duas, e, por outro, com diferenças nas proporções de nulíparas e multíparas, cerca de $60 \%$ de multíparas nos estudos referidos e $48 \%$ no nosso estudo.

Vários estudos referem que o aumento de peso gestacional está diretamente relacionado com o peso do $\mathrm{RN}$, sendo que aumentos de peso inferiores ou superiores ao recomendado estão associados a RN leves para idade gestacional ou grandes para a idade gestacional, respetivamente. ${ }^{3,5,8} \mathrm{~A}$ longo prazo a principal consequência do ganho de peso gestacional excessivo, para a criança, é a obesidade, com todas as implicações e comorbilidades associadas. ${ }^{4}$ Apesar de não se ter verificado associação estatisticamente significativa entre a classe de aumento de peso e o tipo de peso do RN (baixo peso, normal ou macrossómico), verificamos que as grávidas com aumento de peso excessivo têm em média filhos mais pesados do que as grávidas com au- mento de peso adequado ou inferior, o que vem corroborar a evidência anterior.

Como principal limitação deste estudo destaca-se o facto de a maior parte das grávidas que apresentaram complicações durante a gravidez serem habitualmente referenciadas para os cuidados secundários e posteriormente seguidas a nível hospitalar, pelo que foram excluídas do estudo. De facto verificou-se uma baixa incidência de síndromes hipertensivos ( $2 \%$ HTA gestacional e $0,8 \%$ de pré-eclâmpsia) em relação aos $8 \%$ descritos na literatura. ${ }^{13}$ Esta limitação poderá ter comprometido os resultados do nosso estudo, nomeadamente no objetivo de avaliar associação entre aumento de peso na gravidez e complicações obstétricas.

Importa ainda referir o viés de informação, por um lado devido à colheita dos dados, que teve em conta os registos clínicos, por outro lado devido à subjetividade do peso pré-concecional, que foi o reportado pela grávida na primeira consulta de gravidez ou o medido nessa ocasião, sendo esta sempre anterior às 12 semanas de gestação. Relativamente às variações de peso e à sua relação com o IMC pré-concecional e com o peso do $\mathrm{RN}$, os nossos resultados estão globalmente de acordo com a literatura, pelo que não se espera que estes viéses tenham afetado os resultados.

Este estudo teve em conta as recomendações atualmente aceites por instituições internacionais, como o IOM e a Organização Mundial de Saúde que, no entanto, não foram validadas para Portugal, pelo que admitimos que os valores recomendados de aumento de peso possam não ser os mais adequados para a nossa população. Um estudo sueco, realizado entre 1994 e 2004, estabeleceu outros valores recomendados para aumento de peso, baseado na ocorrência de complicações obstétricas e perinatais. Segundo este estudo, mulheres com IMC normal devem aumentar entre 2 e 10 $\mathrm{kg}$, mulheres com excesso de peso menos de $9 \mathrm{~kg}$ e mulheres obesas menos de $6 \mathrm{~kg}$.

A obesidade e o aumento de peso excessivo durante a gravidez são entidades que podem ser corrigidas/prevenidas com mudança dos padrões comportamentais, sendo as ações de educação para a saúde importantes estratégias terapêuticas. ${ }^{4} \mathrm{O}$ médico de família tem um importante papel não só na vigilância da gravidez, mas também na sua preparação. A prevenção da obesidade na mulher em idade fértil é uma medida importante 
para o desenvolvimento de uma gravidez saudável. $\mathrm{Na}$ vigilância da gravidez o médico de família deve estar atento à adequada evolução ponderal nas diferentes fases da gestação.

Uma vez que o aumento excessivo de peso na gravidez pode ser um fator promotor do aumento da prevalência de obesidade na população (quer pela grávida, quer pelo RN), ${ }^{14,15}$ são necessárias recomendações referentes ao aumento de peso durante a gravidez de acordo com o IMC adaptadas à nossa população.

Em conclusão, o IMC pré-concecional elevado está associado a ganho ponderal gestacional superior ao recomendado. Contrariamente às recomendações, as mulheres com maior IMC têm maior aumento de peso.

\section{REFERÊNCIAS BIBLIOGRÁFICAS}

1. Finucane MM, Stevens GA, Cowan MJ, Danaei G, Lin JK, Paciorek CJ, et al. National, regional, and global trends in body-mass index since 1980: systematic analysis of health examination surveys and epidemiological studies with 960 country-years and 9.1 million participants. Lancet 2011 Feb 12; 377 (9765): 557-67.

2. Poínhos R, Franchini B, Afonso C, Correia F, Teixeira V, Moreira P, et al. Alimentação e estilos de vida da população portuguesa: metodologia e resultados preliminares. Alimentação Humana 2009; 15 (3): 43-60.

3. Crane JM, White J, Murphy P, Burrage L, Hutchens D. The effect of gestacional weight gain by body mass index on maternal and neonatal outcomes. J Obstet Gynaecol Can 2009 Jan; 31 (1): 28-35.

4. Rasmussen KM, Yaktine AL, editors. Weight Gain during Pregnancy: Reexamining the Guidelines. Washington, DC: National Academies Press; 2009. Disponível em: http://www.nap.edu/catalog.php?record_id= 12584 [acedido em 11/08/2012].

5. Moura JM,Ayres-de-Campos D. Consequências obstétricas e perinatais da obesidade materna. Acta Obstet Ginecol Port 2010; 4 (4): 193-7.

6. Committee on Nutritional Status During Pregnancy and Lactation, Institute of Medicine. Nutrition During Pregnancy: Part I: Weight Gain, Part II: Nutrient Supplements. Washington, DC: National Academies Press; 1990. Disponível em: http://www.nap.edu/catalog.php? record_id=1451 [acedido em 11/08/2013].

7. Cedergren MI. Optimal gestacional weight gain for body mass index categories. Obstet Gynecol 2007 Oct; 110 (4): 759-64.

8. Mamun AA, Callaway LK, O'Callaghan MJ, Williams GM, Najman JM, Alati R, et al. Associations of maternal pre-pregancy obesity and excess pregnancy weight gains with adverse pregnancy outcomes and length of hospital stay. BMC Pregnancy Childbirth 2011 Sep 6; 11: 62.
9. Chasan-Taber L, Schmidt MD, Pekow P, Sternfeld B, Solomon CG, Markenson $G$. Predictors of excessive and inadequate gestational weight gain in Hispanic women. Obesity 2008 Jul; 16 (7): 1657-66.

10. Olson G, Blackwell SC. Optimization of gestational weight gain in the obese gravida: a review. Obstet Gynecol Clin N Am 2011 Jun; 38 (2): 397-407.

11. DeVader SR, Neeley HL, Myles TD, Leet TL. Evaluation of gestacional weight gain guidelines for women with normal pre-pregnancy body mass index. Obstet Gynecol 2007 Oct; 110 (4): 745-51.

12. Chu SY, Callaghan WM, Bish CL, D'Angelo D. Gestational weight gain by body mass index among US women delivering live births, 2004-2005: fueling future obesity. Am J Obstet Gynecol 2009 Mar; 200 (3): 271-7.

13. Barra S, Cachulo MC, Providência R, Leitão-Marques A. Hipertensão arterial na grávida: o atual estado da arte. Rev Port Cardiol 2012 Jun; 31 (6): 425-32.

14. Wrotniak BH, Shults J, Butts S, Stettler N. Gestational weight gain and risk of overweight in the offspring at age $7 \mathrm{y}$ in a multicenter, multiethnic cohort study. Am J Clin Nutr 2008 Jun; 87 (6): 1818-24.

15. Oken E, Rifas-Shiman SL, Field AE, Frazier AL, Gillman MW. Maternal gestational weight gain and the offspring in adolescence. Obstet Gynecol 2008 Nov; 112 (5): 999-1006.

\section{CONFLITOS DE INTERESSE}

Não existiram fontes externas de financiamento para a realização deste artigo.

Os autores declaram não ter nenhum conflito de interesses relativamente ao presente artigo.

\section{ENDEREÇO PARA CORRESPONDÊNCIA}

Joana Sequeira

Rua D. Manuel Barbuda Vasconcelos N. ${ }^{\circ} 354^{\circ} \mathrm{SE}$

3810-498 Aveiro

joanaseq@gmail.com

Recebido em 17/12/2012

Aceite para publicação em 18/02/2013

Artigo escrito ao abrigo do novo acordo ortográfico. 


\section{ABSTRACT}

\section{WEIGHT GAIN IN PREGNANCY: A RETROSPECTIVE STUDY}

Objectives: To assess weight gain in pregnancy and to test the associations between weight gain in pregnancy and the mode of delivery, parity, obstetric complications and birth weight of the newborn.

Study design: Historical cohort study.

Setting: Vagos Health Center, ACES Baixo Vouga II, Portugal.

Participants: Pregnant women followed in the health center.

Methods: The study sample was composed of women who were pregnant between 2003 and 2010. The variables studied were age, parity, body mass index (BMI) before pregnancy, weight gain during pregnancy, type of delivery, obstetric complications and the birth weight of the newborn. For statistical analysis we used the Chi-Square Test, ANOVA and Least Significant Difference.

Results: We studied 495 women with a mean age of 27 years. At the beginning of pregnancy $35 \%$ had a BMI $\geq 25$ and $46 \%$ had excessive weight gain during pregnancy. Women with excessive weight gain in pregnancy had a higher mean pre-pregnancy BMI than women with adequate weight gain or weight gain lower than recommended $(p<0,001)$. The children of mothers with excessive weight gain had significantly higher birth weights than other newborns $(p=0.024)$. There was no association between weight gain and the type of delivery, obstetric complications or parity.

Conclusions: About one third of women were overweight or obese at the beginning of pregnancy. Most women had excessive weight gain for their BMI. Women with a higher pre-pregnancy BMI were more likely to have excessive weight gain in pregnancy and heavier babies. Studies are required to validate recommendations for weight gain during pregnancy for the Portuguese population.

Key words: Pregnancy; Obesity; Weight gain. 